vegetables. It also appears different from EoE as uncommon foods such as banana, melon, and avocado were capable of invoking symptoms, notably foods more commonly associated with pollen food allergy syndrome. However, the strong association with atopy, the rapid onset of symptoms, and symptoms distinct from dysphagia suggest a local possibly immunologic factor causing an immediate esophageal mucosal response. A careful history of FIRE symptoms should be part of the routine history of patients with eosinophilic esophagitis. Additionally, a history of IgE-mediated food allergies, allergic rhinitis, and pollen food allergy syndrome should be obtained Further investigations will be required to help better understand the frequency and mechanism of this newly described phenomenon.

\section{KEYWORDS}

anaphylaxis, esophagitis, food allergy

\section{ACKNOWLEDGEMENTS}

None.

CONFLICT OF INTEREST

$\mathrm{MH}$ no conflicts, AS no conflicts.
Mark Holbreich ${ }^{1}$ (D) Alex Straumann ${ }^{2}$

\footnotetext{
${ }^{1}$ Allergy and Asthma Consultants, Indianapolis, IN, USA

${ }^{2}$ Swiss EoE Clinics and Research Network, Department of
} Gastroenterology and Hepatology, University Hospital Zurich,

Zurich, Switzerland

Correspondence

Mark Holbreich, Allergy and Asthma Consultants, $8902 \mathrm{~N}$ Meridian Street, Indianapolis, IN, USA. Email: mholbreich@comcast.net

ORCID

Mark Holbreich (ID) https://orcid.org/0000-0002-5902-2584

\section{REFERENCES}

1. Schoepfer AM, Straumann A, Panczak R, et al. Development and validation of a symptom-based activity index for adults with eosinophilic esophagitis. Gastroenterology. 2014;147:1255-1266.

2. Biedermann L, Holbreich M, Atkins D, et al. Food-induced immediate response of the esophagus-A newly identified syndrome in patients with eosinophilic esophagitis. Allergy. 2021 Jan;76(1):339-347.

\title{
BNT162b2 mRNA COVID-19 vaccine induces antibodies of broader cross-reactivity than natural infection, but recognition of mutant viruses is up to 10 -fold reduced
}

To the Editor,

The receptor binding domain (RBD) of SARS-CoV-2 spike (S) glycoprotein, which is involved in virus attachment and cell entry, is the primary target for neutralizing antibodies. ${ }^{1}$ Immunization against full-length $S$ or parts of it may result in more potent and longerlasting antibody responses than natural viral infection. ${ }^{2}$ Therefore, global vaccination programs based on induction of neutralizing antibodies are the most promising strategy for controlling the COVID-19 pandemic.

A worrying factor, however, has been the emerging variants capable to escape immunity produced by vaccination or infection. Three variants have attracted attentions due to their abnormally high rates of propagation: B.1.1.7 (N501Y, D614G); B.1.351 (K417 N, E484 K, N501Y, D614G); and P.1 (K417 N/T, E484 K, N501Y). Limited knowledge of the presence of cross-neutralizing antibodies induced by natural infection or vaccination is a key gap in current understanding of the spread of SARS-CoV-2. It is imperative to determine the impact of these mutations on the responses induced by currently marketed vaccines.

A study has shown that $\mathrm{E} 484 \mathrm{~K}$ mutation is associated with reduced neutralization, by SARS-CoV-2 infection or BNT162b2elicited sera. ${ }^{3}$ Whether reduced neutralization was due to impaired binding was, however, not analyzed. Here, we assessed the presence of such cross-reactive antibodies in convalescent sera and sera from individuals immunized with mRNA-based BNT162b2 vaccine.

To this end, we generated four mutant RBDs:K417 N (RBD 417$)$, E484 K $\left(\mathrm{RBD}_{484}\right), \mathrm{N} 501 \mathrm{Y}\left(\mathrm{RBD}_{501}\right)$, and one triple mutated version with all three mutations $\left(\mathrm{RBD}_{\text {trip }}\right)$ (Figure $\left.1 \mathrm{~A}\right)$. Two of these mutations, $\mathrm{E} 484 \mathrm{~K}$ and N501Y, are localized within the receptor binding motif, ${ }^{4}$ directly interacting with ACE2. Our ELISA results show that binding of convalescent sera was strongly reduced for $\mathrm{RBD}_{417}$ and $\mathrm{RBD}_{501}$ and,

This is an open access article under the terms of the Creative Commons Attribution-NonCommercial-NoDerivs License, which permits use and distribution in any medium, provided the original work is properly cited, the use is non-commercial and no modifications or adaptations are made.

(c) 2021 The Authors. Allergy published by European Academy of Allergy and Clinical Immunology and John Wiley \& Sons Ltd. 
(A)

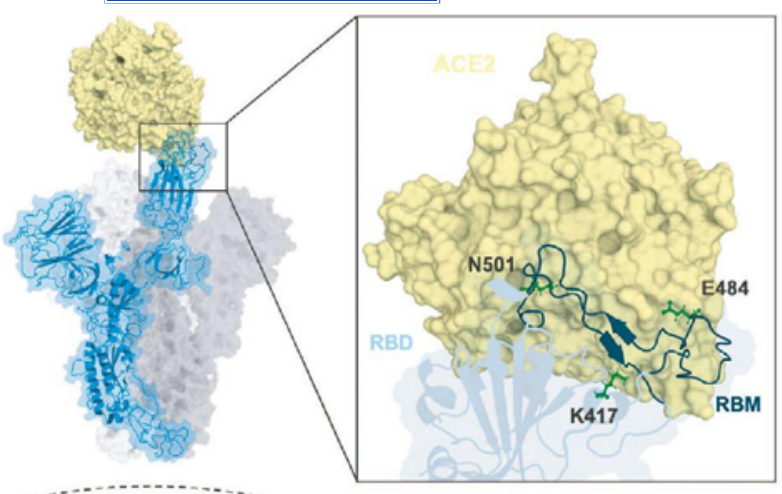

INTRAVIRIUM

(B)
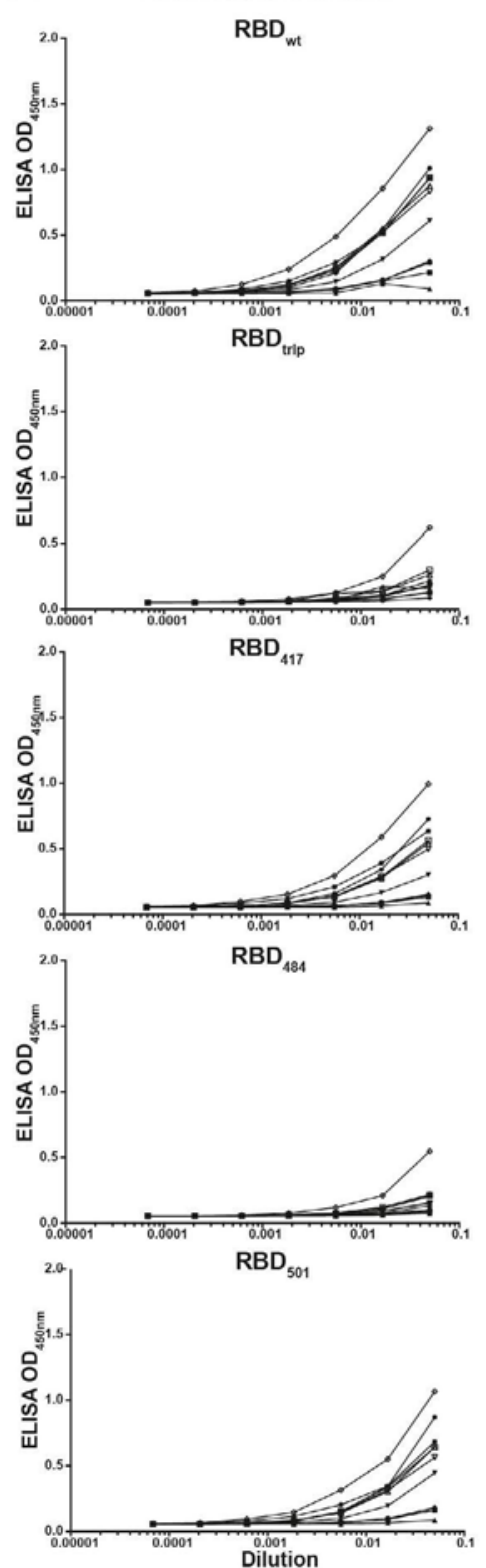

BNT162b2 Vaccinated sera
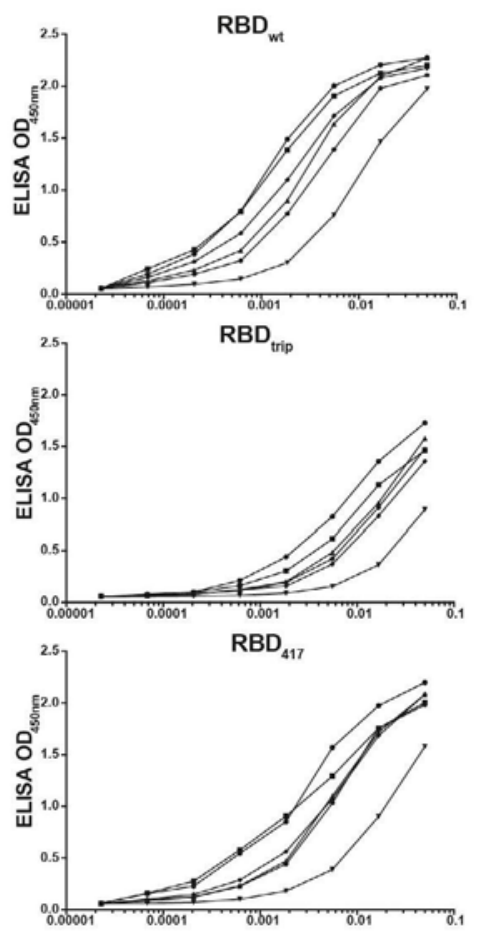

$\mathrm{RBD}_{484}$

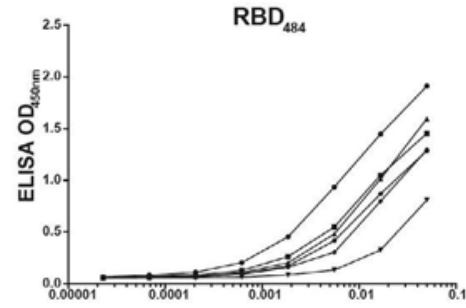

RBD $_{501}$

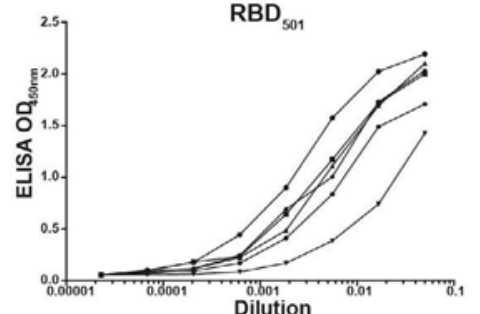

(C) BNT162b2 Vaccinated sera

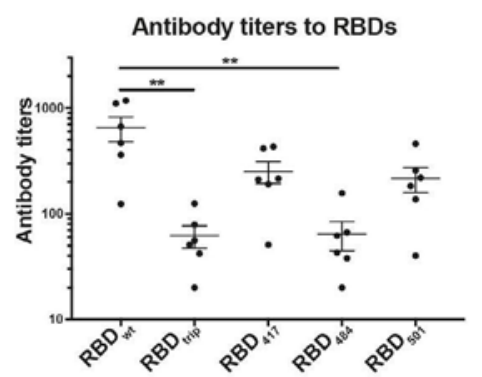

(D) Titer change of mutated to wt RBD

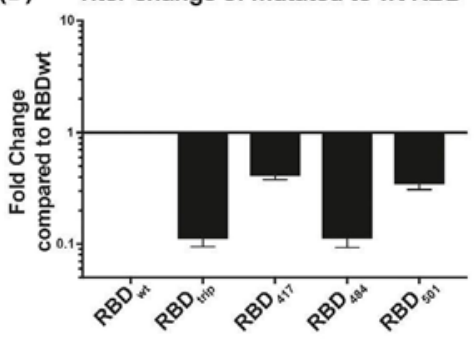

FIGURE 1 Strongly reduced recognition of mutant RBDs by convalescent and BNT162b2 vaccinated human sera. (A) Structure of RBD and location of the individual mutations used in this study (E484 K, K417 N, N501Y, or all 3 mutations combined). (B) Titration on RBDs of sera from convalescent patients (left panel) or from BNT162b2 vaccinated individuals (right panel). (C) Antibody titers (OD50) of sera from 6 BNT162b2 vaccinated individuals on RBDs. (D) Fold reduction of mutant RBDs compared to wild-type RBD-recognition by sera of BNT162b2 vaccinated individuals 
(A)

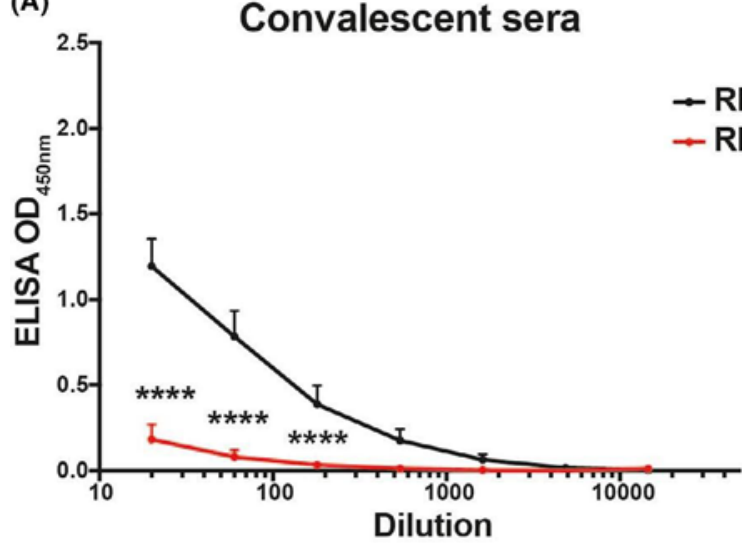

(B)

\section{BNT162b2 vaccinated sera}

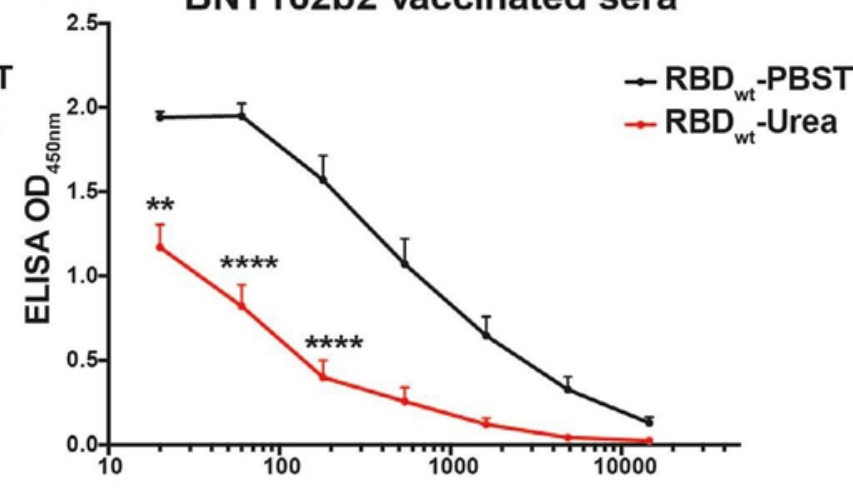

(C)

Avidity index--BNT162b2 Vaccinated sera

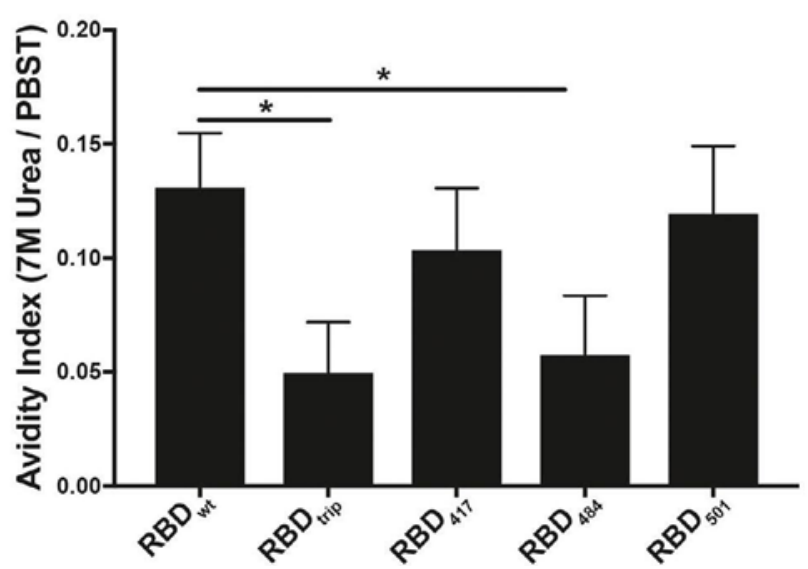

FIGURE 2 Increased avidity of BNT162b2-induced antibodies compared to antibodies induced by infection. (A, B) Average recognition of $\mathrm{RBD}_{\mathrm{wt}}$ by convalescent (A) or vaccine-induced sera (B) by avidity ELISA. Samples washed with PBS-Tween (black) and $7 \mathrm{M}$ urea (red). (C) Avidity indexes are shown for recognition of mutant RBDs by vaccine-induced sera. These indexes are calculated by dividing the area under the curve (AUC) of urea washed samples by AUC of PBS-Tween washed samples. Note that recognition of mutant RBDs by convalescent sera was too low to calculate a meaningful avidity index

however, essentially abolished for $\mathrm{RBD}_{484}$ and $\mathrm{RBD}_{\text {trip }}$ (Figure $1 \mathrm{~B}$, left). In contrast, BNT162b2-elicited antibodies exhibited only weakly reduced binding to $\mathrm{RBD}_{417}$ and $\mathrm{RBD}_{501}$ (2.5-3-fold), but 10 -fold reduced binding to $R_{B D} D_{484}$ and $R B D_{\text {trip }}$ (Figure $1 \mathrm{~B}$, right). Figure $1 \mathrm{C}$ summarizes the antibody titers and Figure $1 D$ quantifies the reduced binding of vaccine-induced sera to the mutant RBDs compared to $R_{B D}$ (wildtype $\mathrm{RBD}$ ). Interestingly, the $\mathrm{E} 484 \mathrm{~K}$ mutation was equally potent at reducing antibody binding as in $\mathrm{RBD}_{\text {trip }}$, indicating that the mutation E484 $\mathrm{K}$ is particularly problematic, perhaps because it involves a change from positive to negative charge. This is consistent with recent data showing less neutralization titer against a variant containing E484 K mutation, however, only with 1.41-fold reduction. ${ }^{5}$

We performed assays to estimate the antibodies' avidity for RBD and mutants. Interestingly, virus-induced antibodies were of limited avidity for $\mathrm{RBD}_{\mathrm{wt}}$, and binding to mutant RBDs was essentially abolished with $7 \mathrm{M}$ urea wash, indicating that the antibodies binding to mutant RBDs were all of low avidity (Figure 2A shows results for $\mathrm{RBD}_{\mathrm{wt}}$ ). In contrast, BNT162b2-induced antibodies were of significantly higher avidity (Figure 2B). In addition, there was some residual binding to mutant RBDs, indicating, however, overall low avidity as well (Figure $2 \mathrm{C}$ ). The avidity index allows to quantify the loss in binding caused by the $7 \mathrm{M}$ urea wash and therefore reflects the "quality" of the antibodies. Indeed, the avidity index of vaccine-induced antibodies is much higher for $R B D_{w t}$ and mutants than those induced by infection. This reduced affinity of antibodies induced by infection is consistent with the notion that individual RBDs are spaced by $25 \mathrm{~nm}$ on SARS-CoV-2, too large for inducing optimal antibodies. ${ }^{6}$

In conclusion, BNT162b2-induced antibodies recognize mutant RBDs better than those by natural infection. Recognition may, however, be 10-fold reduced for the variants B.1.351/P.1, suggesting that development of a new vaccine may be warranted. E484 $\mathrm{K}$ mutation is shown here to be a key hurdle for immune recognition. Hence, monoclonal antibody therapy and serological assays based on wildtype sequence may therefore be seriously impaired. 


\section{KEYWORDS}

COVID, SARS-CoV, vaccines

\section{FUNDING INFORMATION}

The work was supported by Saiba AG, the Swiss National Science Foundation (SNF grants 31003A 149925 and 310030-179459) and the Inselspital, Bern, Switzerland.

\section{ACKNOWLEDGEMENTS}

We thank Marianne Zwicker for production of mutant RBDs.

\section{CONFLICT OF INTEREST}

M. F. Bachmann is a board member of Saiba AG, involved in the development of RBD-CuMV a vaccine against COVID-19. All other authors declare no conflict of interest.

\section{Xinyue Chang 1,2 (D) Gilles Sousa Augusto ${ }^{1,2,3}$ (D) Xuelan $\operatorname{Liu}^{1,4}$ \\ Thomas M. Kündig ${ }^{5}$ Monique Vogel ${ }^{1,2}$ (1) \\ Mona O. Mohsen ${ }^{1,2}$ Martin F. Bachmann ${ }^{1,2,3,4}$ (D)}

${ }^{1}$ Immunology, RIA, University of Bern, Bern, Switzerland ${ }^{2}$ Department of BioMedical Research, University of Bern, Bern,

Switzerland

${ }^{3}$ Nuffield Department of Medicine, Centre for Cellular and Molecular Physiology (CCMP), The Jenner Institute, University of Oxford, Oxford, UK

${ }^{4}$ International Immunology Centre, Anhui Agricultural University, Hefei, China

${ }^{5}$ University Hospital Zürich, Zürich, Switzerland

\section{Correspondence}

Martin F. Bachmann and Mona O. Mohsen, University Clinic of Rheumatology and Immunology, University Hospital Bern, Salihaus 2, CH-3010 Bern, Switzerland. Emails: martin.bachmann@dbmr.unibe.ch (MFB); mona.mohsen@ dbmr.unibe.ch (MOM)

Mohsen and Bachmann equal contribution.

ORCID

Xinyue Chang (D) https://orcid.org/0000-0001-7027-3889

Gilles Sousa Augusto (iD https://orcid.org/0000-0001-6509-0148

Monique Vogel (D) https://orcid.org/0000-0002-5219-4033

Martin F. Bachmann (D) https://orcid.org/0000-0003-4370-2099

\section{REFERENCES}

1. Tai W, He L, Zhang X, et al. Characterization of the receptor-binding domain (RBD) of 2019 novel coronavirus: implication for development of RBD protein as a viral attachment inhibitor and vaccine. Cell Mol Immunol. 2020;17:613-620.

2. Tay MZ, Poh CM, Renia L, MacAry PA, Ng LFP. The trinity of COVID-19: immunity, inflammation and intervention. Nat Rev Immunol. 2020;20:363-374.

3. Dejnirattisai W, Zhou D, Supasa P, Ren J, Stuart DI, Screaton GR. Article antibody evasion by the P.1 strain of SARSCoV-2 antibody evasion by the P.1 strain of SARS-CoV-2. Cell. 2021;184:1-16.

4. Greaney AJ, Starr TN, Gilchuk P, et al. Complete mapping of mutations to the SARS-CoV-2 spike receptor-binding domain that escape antibody recognition. Cell Host Microbe. 2021;29:44.

5. Xie X, Liu Y, Liu J, et al. Neutralization of SARS-CoV-2 spike 69/70 deletion, E484K and N501Y variants by BNT162b2 vaccine-elicited sera. Nat Med. 2021;27:620-621.

6. Bachmann MF, Mohsen MO, Zha L, Vogel M, Speiser DE. SARSCoV-2 structural features may explain limited neutralizing-antibody responses. NPJ Vaccines. 2021;6(1):2.

\section{Asia-Pacific perspectives on the COVID-19 pandemic}

To the Editor,

Coronavirus disease 2019 (COVID-19) has affected over ten millions of people globally since the World Health Organization declared it a pandemic on 11 March 2020. ${ }^{1}$ The Asia-Pacific is a diverse geographical region with different health care systems and levels of access to specialist services. This survey was commissioned by the Asia Pacific Association of Allergy Asthma and Clinical Immunology (APAAACI) Task Force on COVID-19 with the premise to understand the epidemiology, ${ }^{2}$ clinical profile (including severity and risk factors), ${ }^{3,4}$ therapeutics/access to clinical trials, ${ }^{5}$ impact on clinical immunology and allergy services/therapeutics, ${ }^{6}$ occupational health and mental well-being (supporting information S1, S2) of healthcare providers in the region.

A questionnaire comprising 44 questions was electronically sent out to 15 member countries of APAAACl using Survey Monkey (B) on $8^{\text {th }}$ May 2020. The questionnaire was sent out to member societies through their presidents who responded based on the prevailing COVID-19 situation during the survey period in each 